\title{
Effective action of three-dimensional extended supersymmetric matter on gauge superfield background
}

\author{
I.L. Buchbinder ${ }^{\dagger}$, N.G. Pletnev ${ }^{\ddagger}$, I.B. Samsonov ${ }^{\star}$ \\ ${ }^{\dagger}$ Department of Theoretical Physics, Tomsk State Pedagogical University, \\ 634061 Tomsk, Russia, email: joseph@tspu.edu.ru \\ ${ }^{\ddagger}$ Department of Theoretical Physics, Institute of Mathematics, 630090 Novosibirsk, Russia \\ email: pletnev@math.nsc.ru \\ ${ }^{\star}$ Laboratory of Mathematical Physics, Tomsk Polytechnic University, 634050 Tomsk, Russia \\ email: samsonov@mph.phtd.tpu.ru
}

\begin{abstract}
We study the low-energy effective actions for gauge superfields induced by quan$\operatorname{tum} \mathcal{N}=2$ and $\mathcal{N}=4$ supersymmetric matter fields in three-dimensional Minkowski space. Analyzing the superconformal invariants in the $\mathcal{N}=2$ superspace we propose a general form of the $\mathcal{N}=2$ gauge invariant and superconformal effective action. The leading terms in this action are fixed by the symmetry up to the coefficients while the higher order terms with respect to the Maxwell field strength are found up to one arbitrary function of quasi-primary $\mathcal{N}=2$ superfields constructed from the superfield strength and its covariant spinor derivatives. Then we find this function and the coefficients by direct quantum computations in the $\mathcal{N}=2$ superspace. The effective action of $\mathcal{N}=4$ gauge multiplet is obtained by generalizing the $\mathcal{N}=2$ effective action.
\end{abstract}




\section{Introduction}

Modern interest to three-dimensional supergauge models with extended supersymmetry is motivated mainly by recent progress in constructing and studying the field theories describing the worldvolume degrees of freedom of M2 branes. Such models are usually referred to as the Bagger-Lambert-Gustavsson (BLG) [1] and Aharony-Bergman-JafferisMaldacena (ABJM) [2] theories which are the superconformal Chern-Simons-matter models with $\mathcal{N}=8$ and $\mathcal{N}=6$ supersymmetry, respectively. Since the superconformal symmetry is preserved on the quantum level, these theories are dual to the superstring theory on the corresponding background within the $\mathrm{AdS}_{4} / \mathrm{CFT}_{3}$ correspondences.

One of the general problems for the BLG and ABJM models is to study the effective action which would describe an effective quantum dynamics of M2 branes. In particular, such effective actions receive contributions in the gauge field sector induced by quantum matter fields which can be studied independently of the other contributions. A good starting point for understanding this general issue is the effective action for the Abelian gauge superfield induced by quantum matter superfields.

In the present paper we explore the three-dimensional supersymmetric Euler-Heisenberg-type effective action which appears as a result of one-loop contributions from quantum supersymmetric matter. This problem is interesting not only from the point of view of BLG and ABJM models, but also as a part of the effective action in the three-dimensional supersymmetric electrodynamics. In the non-supersymmetric case this problem was studied in [3], but superspace analysis has never been done (c.f. the effective action in the fourdimensional supersymmetric electrodynamics which was studied in superspace in $[4,5,6]$ ). In the present paper we fill this gap by deriving the Euler-Heisenberg effective actions for the model of $\mathcal{N}=2$ chiral superfield and $\mathcal{N}=4$ charged hypermultiplet interacting with the background gauge superfields.

In our work we employ the $\mathcal{N}=2, d=3$ superspace approach which is similar to the $\mathcal{N}=1, d=4$ superspace. In particular, the $\mathcal{N}=2, d=3$ chiral and vector multiplets appear by dimensional reduction from the four-dimensional $\mathcal{N}=1$ supersymmetric ones while the hypermultiplet and the $\mathcal{N}=4$ vector multiplet in three dimensional Minkowski space originate from the $\mathcal{N}=2, d=4$ hypermultiplet and gauge superfield, respectively. We consider the background $\mathcal{N}=2, d=3$ gauge superfield constrained by $D_{\alpha} W_{\beta}=$ $D_{(\alpha} W_{\beta)}=$ const, where $W_{\alpha}$ is the superfield strength. In components, this constraint corresponds to the constant Maxwell field strength, $F_{m n}=$ const.

As soon as the classical action of the chiral superfield in the background gauge superfield is superconformal, the resulting effective action should be superconformal as well. We show that the gauge and superconformal invariance restrict the functional form of the leading terms in the effective action uniquely, up to coefficients, while the higher order terms with respect to the Maxwell field strength are encoded in a single arbitrary function of one superconformal quasi-primary superfield. Then we find this function as well as the coefficients by direct quantum computations in the $\mathcal{N}=2, d=3$ superspace. A straightforward generalization of these results to the $\mathcal{N}=4$ case leads to the effective action of the $\mathcal{N}=4$ charged hypermultiplet interacting with background gauge superfield. 
The paper is organized as follows. We begin Section 2 with a short review of the chiral superfield model in the $\mathcal{N}=2$ superspace and specify the constraints on the background gauge superfield under considerations. Then we discuss general structure of the gaugesuperfield-dependent $\mathcal{N}=2$ supersymmetric effective action subject to the constraints of gauge and superconformal invariance. In Section 3 we compute the one-loop effective actions in the models of $\mathcal{N}=2$ chiral superfield interacting with the background gauge superfield as well as for the $\mathcal{N}=4$ charged hypermultiplet using the Fock-Schwinger's proper-time technique in the $\mathcal{N}=2$ superspace. In the last section we discuss the obtained results and their possible generalizations. Appendix A contains basic formulae concerning the $\mathcal{N}=2, d=3$ superspace in our conventions. In Appendix B we consider a representation of the superconformal group on the superfields in the $\mathcal{N}=2$ superspace.

\section{General structure of superconformal effective ac- tion in $\mathcal{N}=2$ superspace}

\subsection{Classical action of chiral superfield interacting with the gauge superfield}

In this subsection we review some features of the $\mathcal{N}=2, d=3$ chiral and gauge superfield models which will be used in the next sections. Our conventions for the $\mathcal{N}=2$ superspace are collected in the Appendix A.

Let us consider a classical action for the chiral superfield $Q$ interacting with the Abelian background gauge superfield $V$,

$$
S_{\mathcal{N}=2}=-\int d^{3} x d^{4} \theta \bar{Q} e^{2 V} Q
$$

which is invariant under the following gauge transformations

$$
Q \rightarrow e^{i \Lambda} Q, \quad \bar{Q} \rightarrow \bar{Q} e^{-i \bar{\Lambda}}, \quad e^{2 V} \rightarrow e^{i \bar{\Lambda}} e^{2 V} e^{-i \Lambda},
$$

with $\Lambda$ and $\bar{\Lambda}$ being (anti)chiral superfield gauge parameters. The chiral multiplet consists of the complex scalar $f$, complex spinor $\psi_{\alpha}$ and complex auxiliary scalar $F$,

$$
Q=f+\theta^{\alpha} \psi_{\alpha}+\theta^{2} F+i \theta^{\alpha} \bar{\theta}^{\beta} \partial_{\alpha \beta} f+\frac{i}{2} \theta^{2} \bar{\theta}^{\alpha} \partial_{\alpha \beta} \psi^{\beta}+\frac{1}{4} \theta^{2} \bar{\theta}^{2} \square f .
$$

The vector multiplet in three dimensions is built from one real scalar $\phi$, one complex spinor $\lambda_{\alpha}$, one vector field $A_{\alpha \beta}=\gamma_{\alpha \beta}^{m} A_{m}$ and one real auxiliary scalar $D$. In the Wess-Zumino gauge the component decomposition for $V$ is given by

$$
V=\theta^{\alpha} \bar{\theta}^{\beta} A_{\alpha \beta}+i \theta^{\alpha} \bar{\theta}_{\alpha} \phi+i \theta^{2} \bar{\theta}^{\alpha} \bar{\lambda}_{\alpha}-i \bar{\theta}^{2} \theta^{\alpha} \lambda_{\alpha}+\theta^{2} \bar{\theta}^{2} D .
$$

It is important to specify the background gauge superfield under considerations. In general, the vector multiplet arises within standard geometric approach based on covariantization of the flat superspace derivatives,

$$
D_{\alpha} \rightarrow \nabla_{\alpha}=D_{\alpha}+\mathbf{A}_{\alpha}, \quad \bar{D}_{\alpha} \rightarrow \bar{\nabla}_{\alpha}=\bar{D}_{\alpha}+\overline{\mathbf{A}}_{\alpha}, \quad \partial_{m} \rightarrow \nabla_{m}=\partial_{m}+\mathbf{A}_{m},
$$


where the following superfield constraints are imposed $[7,8,9]$

$$
\begin{aligned}
\left\{\nabla_{\alpha}, \bar{\nabla}_{\beta}\right\} & =-2 i\left(\gamma^{m}\right)_{\alpha \beta} \nabla_{m}+2 i \varepsilon_{\alpha \beta} G \\
{\left[\nabla_{\alpha}, \nabla_{m}\right] } & =-\left(\gamma_{m}\right)_{\alpha \beta} \bar{W}^{\beta}, \quad\left[\bar{\nabla}_{\alpha}, \nabla_{m}\right]=\left(\gamma_{m}\right)_{\alpha \beta} W^{\beta} \\
{\left[\nabla_{m}, \nabla_{n}\right] } & =i \mathbf{F}_{m n} .
\end{aligned}
$$

The superfield strengths in the rhs in (2.6)-(2.8) satisfy the following reality properties

$$
G^{*}=G, \quad\left(W^{\alpha}\right)^{*}=\bar{W}^{\alpha}, \quad\left(\mathbf{F}_{m n}\right)^{*}=\mathbf{F}_{m n} .
$$

As usual, there are many Bianchi identities for these superfield strengths which are important for studies of the effective action and quantization. In particular, the superfield strengths $W_{\alpha}$ and $\bar{W}_{\alpha}$ are (anti)chiral,

$$
\bar{D}_{\alpha} W_{\beta}=0, \quad D_{\alpha} \bar{W}_{\beta}=0
$$

and obey

$$
D^{\alpha} W_{\alpha}=\bar{D}^{\alpha} \bar{W}_{\alpha}
$$

An important feature of the $\mathcal{N}=2, d=3$ superspace formulation of the gauge multiplet is that the superfield strengths $W_{\alpha}, \bar{W}_{\alpha}$ are expressed in terms of the scalar superfield strength $G$,

$$
W_{\alpha}=\bar{D}_{\alpha} G, \quad \bar{W}_{\alpha}=D_{\alpha} G,
$$

subject to the following constraints

$$
D^{2} G=0, \quad \bar{D}^{2} G=0 .
$$

These constraints mean that $G$ is a linear superfield. There are also the following useful relations among the superfield strengths

$$
\begin{aligned}
& D_{(\alpha} W_{\beta)}-\bar{D}_{(\alpha} \bar{W}_{\beta)}=\varepsilon^{m n p}\left(\gamma_{p}\right)_{\alpha \beta} \mathbf{F}_{m n}, \\
& D_{(\alpha} W_{\beta)}+\bar{D}_{(\alpha} \bar{W}_{\beta)}=-2 i \partial_{\alpha \beta} G .
\end{aligned}
$$

In the Abelian case the gauge connections for covariant spinor derivatives in (2.5) can be expressed in terms of one real gauge superfield $V$,

$$
\nabla_{\alpha}=e^{-2 V} D_{\alpha} e^{2 V}=D_{\alpha}+2 D_{\alpha} V, \quad \bar{\nabla}_{\alpha}=\bar{D}_{\alpha} .
$$

As a consequence of the algebra $(2.6,2.7)$, the superfield strengths are given by

$$
G=\frac{i}{2} \bar{D}^{\alpha} D_{\alpha} V, \quad W_{\alpha}=-\frac{i}{4} \bar{D}^{2} D_{\alpha} V, \quad \bar{W}_{\alpha}=-\frac{i}{4} D^{2} \bar{D}_{\alpha} V .
$$

Substituting (2.4) into (2.17) we find the component structure of the superfield strengths, in particular,

$$
G=-\phi+\theta^{\alpha} \bar{\lambda}_{\alpha}-\bar{\theta}^{\alpha} \lambda_{\alpha}+\frac{1}{2} \theta^{\alpha} \bar{\theta}^{\beta} f_{\alpha \beta}-2 i \theta^{\alpha} \bar{\theta}_{\alpha} D+\ldots,
$$

where $f_{\alpha \beta}=\partial_{\alpha}^{\rho} A_{\beta \rho}+\partial_{\beta}^{\rho} A_{\alpha \rho}$ and dots stand for the terms with derivatives of the fields.

Now we specify the constraints on the background gauge superfield under considerations: 
i) The gauge superfield obeys the $\mathcal{N}=2$ supersymmetric free Maxwell equations,

$$
D^{\alpha} W_{\alpha}=0, \quad \bar{D}^{\alpha} \bar{W}_{\alpha}=0 .
$$

ii) Within the derivative expansion of the effective action we look for the leading terms without space-time derivatives of the gauge superfields. Such a long-wave approximation is effectively taken into account by considering the constant background,

$$
\partial_{m} G=0, \quad \partial_{m} W_{\alpha}=0, \quad \partial_{m} \bar{W}_{\alpha}=0 .
$$

This approximation suffices to study the Euler-Heisenberg-type effective action which is induced by the $\mathcal{N}=2$ supersymmetric quantum matter fields.

\subsection{Superconformal invariance and the effective action}

In this subsection we analyse the general structure of the effective action in the model (2.1) employing the constraints imposed by the gauge and superconformal invariance. Similar analysis for the $\mathcal{N}=2, d=4$ superconformal models [10] appeared very useful because it helped to construct an off-shell extension of the terms in the gauge superfield effective action computed in the on-shell approximation. Here we will follow similar lines using the realization of the superconformal group in the $\mathcal{N}=2, d=3$ superspace developed in [11] which is a three-dimensional extension of the general method described in [12].

In general, the effective Lagrangian depends on the gauge superfield $V$, its superfield strengths $G, W_{\alpha}, \bar{W}_{\alpha}$ and their derivatives. The only gauge invariant term with explicit dependence on the gauge superfield $V$ and which cannot be rewritten in terms of the superfield strengths is the Chern-Simons term $[8,9,13]$,

$$
S_{\mathrm{CS}}=\frac{k}{2 \pi} \int d^{3} x d^{4} \theta V G=\frac{k}{2 \pi} \int d^{3} x\left(\frac{1}{2} \varepsilon^{m n p} A_{m} \partial_{n} A_{p}+i \lambda^{\alpha} \bar{\lambda}_{\alpha}-2 \phi D\right),
$$

where $k$ is the Chern-Simons level. All other terms in the effective Lagrangian depend only on the superfield strengths and their derivatives.

Recall that we restricted ourself to the long-wave approximation (2.20) which means that we omit all terms with space-time derivatives of superfields, but the covariant spinor derivatives can appear in the effective Lagrangian. In this approximation there is very limited number of building blocks, i.e., the superfield combinations which the effective action can depend on. First of all, it depends on the superfield strength $G$ as well as on $W_{\alpha}$ and $\bar{W}_{\alpha}$ which involve first covariant spinor derivatives of $G,(2.12)$. Next, there are the objects with two covariant spinor derivatives of $G$,

$$
N_{\alpha \beta} \equiv D_{(\alpha} W_{\beta)}, \quad \bar{N}_{\alpha \beta} \equiv-\left(N_{\alpha \beta}\right)^{*}=\bar{D}_{(\alpha} \bar{W}_{\beta)} .
$$

Note that it is sufficient to consider the objects (2.22) with symmetryzed spinor indices since $D_{[\alpha} W_{\beta]}=\frac{1}{2} \varepsilon_{\alpha \beta} D^{\gamma} W_{\gamma}=0$ for the considered background (2.19). Note also that owing to the identity (2.15), $\bar{N}_{\alpha \beta}$ coincides with $N_{\alpha \beta}$ up to a sign,

$$
N_{\alpha \beta}=-\bar{N}_{\alpha \beta} \text {, }
$$


when $\partial_{m} U=0$. Finally, it is clear that any further spinor derivatives of the superfield strengths vanish in the long-wave approximation (2.20), e.g.,

$$
\bar{D}_{\alpha} D_{\beta} W_{\gamma}=-2 i \partial_{\alpha \beta} W_{\gamma}=0, \quad D^{2} W_{\alpha}=-4 i \partial_{\alpha \beta} \bar{W}^{\beta}=0 \text {. }
$$

We conclude that the general structure of the gauge invariant effective action is given by

$$
\Gamma_{\mathcal{N}=2}=\int d^{3} x d^{4} \theta\left[c_{0} V G+\mathcal{L}_{\text {eff }}\left(G, W_{\alpha}, \bar{W}_{\alpha}, N_{\alpha \beta}\right)\right],
$$

where $c_{0}$ is an arbitrary coefficient and $\mathcal{L}_{\text {eff }}$ is an effective Lagrangian being a real scalar superfield. Further restrictions on the structure of the function $\mathcal{L}_{\text {eff }}$ come from the requirement of the superconformal invariance.

As a warming up exercise we check the superconformal invariance of the classical action (2.1). Indeed, using the explicit realization of the superconformal group in the $\mathcal{N}=2$ superspace given in the Appendix B, we consider the superconformal transformations of the gauge and matter superfields,

$$
\delta_{\mathrm{sc}} V=\xi V, \quad \delta_{\mathrm{sc}} Q=(\sigma / 2+\xi) Q, \quad \delta_{\mathrm{sc}} \bar{Q}=(\bar{\sigma} / 2+\xi) \bar{Q},
$$

where $\xi$ is a superconformal Killing vector (B.10) and $\sigma, \bar{\sigma}$ are (anti)chiral superfields constructed from the parameters of the superconformal transformations, (B.20,B.21). The superconformal variation of the Lagrangian in (2.1) is

$$
\delta_{\mathrm{sc}}\left(\bar{Q} e^{2 V} Q\right)=(\rho+\xi)\left(\bar{Q} e^{2 V} Q\right)
$$

where $\rho=\frac{1}{2}(\sigma+\bar{\sigma})$ is given in (B.17). Equation (2.27) shows that $\bar{Q} e^{2 V} Q$ is a quasiprimary scalar superfield with conformal weight $l=+1$. Hence, according to (B.26), the action (2.1) is invariant under the superconformal transformations, $\delta_{\mathrm{sc}} S_{\mathcal{N}=2}=0$.

Of course, the Chern-Simons action (2.21) is superconformal as well. To show this, we derive the superconformal transformation of the superfield strength $G$ with the help of $(2.17,2.26)$ and (B.15,B.16),

$$
\delta_{\mathrm{sc}} G=(\rho+\xi) G,
$$

i.e., $G$ is a quasi-primary superfield. Using (B.26) we immediately find

$$
\delta_{\mathrm{sc}} S_{\mathrm{CS}}=\frac{k}{2 \pi} \int d^{3} x d^{4} \theta(\rho+\xi) V G=0 .
$$

Hence, the superconformal invariance imposes only constraints on the function $\mathcal{L}_{\text {eff }}$ in $(2.25)$.

In general, the effective Lagrangian contains the effective potential term $\mathcal{F}(G)$,

$$
\mathcal{L}_{\text {eff }}=\mathcal{F}(G)+\tilde{\mathcal{L}}_{\text {eff }}\left(G, W_{\alpha}, \bar{W}_{\alpha}, N_{\alpha \beta}\right)
$$

where $\mathcal{F}(G)$ is a holomorphic function of $G$ only while $\tilde{\mathcal{L}}_{\text {eff }}$ takes into account the superfield strength with covariant spinor derivatives. The superconformal invariance restricts the 
form of the effective potential $\mathcal{F}(G)$ uniquely, up to a constant. Indeed, the general condition of superconformal invariance (B.27) applied to the effective potential reads

$$
\delta_{\mathrm{sc}} \mathcal{F}(G)=(\rho+\xi) \mathcal{F}(G)+\sigma \mathcal{K}(G)+\bar{\sigma} \overline{\mathcal{K}}(G),
$$

where the function $\mathcal{K}(G)$ should be linear,

$$
D^{2} \overline{\mathcal{K}}(G)=\bar{D}^{2} \mathcal{K}(G)=0 \quad \Rightarrow \quad \mathcal{K}(G)=\alpha+\beta G, \quad \overline{\mathcal{K}}(G)=\bar{\alpha}+\bar{\beta} G
$$

with $\alpha$ and $\beta$ being some (complex) constants. Up to the terms vanishing under integral over full $\mathcal{N}=2$ superspace, the general solution of $(2.31)$ is given by ${ }^{1}$

$$
\mathcal{F}(G)=c_{1} G \ln G,
$$

where $c_{1}$ is some constant. This effective potential is responsible for a superconformal generalization of the Maxwell term in its component decomposition,

$$
\int d^{3} x d^{4} \theta G \ln G=\frac{1}{8} \int d^{3} x \frac{1}{\phi} F^{m n} F_{m n}+\ldots,
$$

where dots stand for other component terms. Note that the Lagrangian (2.33) being considered in the $\mathcal{N}=1, d=4$ superspace is responsible for the classical action of the improved tensor multiplet model [12].

It is much more difficult to make general analysis of the admissible form of the function $\tilde{\mathcal{L}}_{\text {eff }}$ in (2.30) subject to the superconformal invariance of the corresponding action. The problem is that the superfields $W_{\alpha}, \bar{W}_{\alpha}$ and $N_{\alpha \beta}$ are not quasi-primary, e.g.,

$$
\delta_{\mathrm{sc}} W_{\alpha}=\left(\frac{1}{2} \rho+\sigma+\xi\right) W_{\alpha}+\omega_{\alpha \beta} W^{\beta}+\left(\bar{D}_{\alpha} \rho\right) G
$$

where $\omega_{\alpha \beta}=\bar{D}_{(\alpha} \bar{\xi}_{\beta)}=-D_{(\alpha} \xi_{\beta)}$ are the parameters of 'local' Lorentz transformations. Equation (2.35) shows that $W_{\alpha}$ transforms inhomogeneously because of the last term in (2.35). This is a new feature of three-dimensional supergauge models as compared to the $\mathcal{N}=1, d=4$ ones in which the superfield strengths are chiral quasi-primary, $[12,14,15]$. Therefore the superfields $W_{\alpha}$ and $\bar{W}_{\alpha}$ are rather inconvenient for constructing superconformal actions and we are forced to introduce the following quasi-primary superfields ${ }^{2}$

$$
\Psi=\frac{i}{G} \bar{D}^{\alpha} D_{\alpha} \ln G, \quad \Omega^{2}=\frac{1}{8}\left(\frac{1}{G} \bar{D}^{\alpha} D_{\alpha}\right)^{2} \ln G .
$$

Indeed, using (2.28) and the relations (B.15,B.16) one can readily check that both these superfields are quasi-primary with zeroth scaling dimension,

$$
\delta_{\mathrm{sc}} \Psi=\xi \Psi, \quad \delta_{\mathrm{sc}} \Omega^{2}=\xi \Omega^{2} .
$$

\footnotetext{
${ }^{1}$ To be more precise, we have to use a dimensionless combination $G / \mu$ under the logarithm in (2.33) where $\mu$ is some scale. However, this parameter is spurious and drops out completely in the component field formulation. Therefore we omit this parameter everywhere for brevity.

${ }^{2}$ In principle, the coefficients in (2.36) are arbitrary, but we fix them in such a way that the effective action (3.51) computed in the next section gets simple form.
} 
This allows us to construct a superconformal action with these superfields,

$$
S_{1}=\int d^{3} x d^{4} \theta G \mathcal{U}\left(\Psi, \Omega^{2}\right), \quad \delta_{\mathrm{sc}} S_{1}=0,
$$

where $\mathcal{U}\left(\Psi, \Omega^{2}\right)$ is an arbitrary function.

Neither the gauge invariance nor the superconformal symmetry impose any restrictions on possible form of the function $\mathcal{U}\left(\Psi, \Omega^{2}\right)$ in (2.38). However, for the background gauge superfield under considerations $(2.19,2.20)$ the form of this functions can be further reduced. Indeed, for such a background there are the following equivalent representations for $\Psi$ and $\Omega^{2}$,

$$
\begin{aligned}
\Psi & =-i \frac{W^{\alpha} \bar{W}_{\alpha}}{G^{3}} \\
\Omega^{2} & =\frac{1}{8} \frac{N_{\beta}^{\alpha} N_{\alpha}^{\beta}}{G^{4}}+\frac{3}{4} \frac{N^{\alpha \beta} W_{\alpha} \bar{W}_{\beta}}{G^{5}}+\frac{3}{4} \frac{W^{2} \bar{W}^{2}}{G^{6}} .
\end{aligned}
$$

Owing to the odd statistics of superfield strengths $W_{\alpha}$ and $\bar{W}_{\alpha}$, the power expansion of $\mathcal{U}\left(\Psi, \Omega^{2}\right)$ over $\Psi$ terminates at the second order,

$$
\mathcal{U}\left(\Psi, \Omega^{2}\right)=\mathcal{U}_{0}\left(\Omega^{2}\right)+\Psi \mathcal{U}_{1}\left(\Omega^{2}\right)+\Psi^{2} \mathcal{U}_{2}\left(\Omega^{2}\right)
$$

Under the integral over $\mathcal{N}=2$ superspace the first two terms in the rhs of (2.41) can be brought to the form of the last term,

$$
\int d^{3} x d^{4} \theta G\left[\mathcal{U}_{0}\left(\Omega^{2}\right)+\Psi \mathcal{U}_{1}\left(\Omega^{2}\right)\right]=\int d^{3} x d^{4} \theta G \Psi^{2} \tilde{\mathcal{U}}_{2}\left(\Omega^{2}\right),
$$

where $\tilde{\mathcal{U}}_{2}$ is some function. Indeed, to check (2.42) one has to take the covariant spinor derivatives from $N_{\alpha \beta}=D_{\alpha} W_{\beta}$ in (2.40) and integrate them by parts. Note that these derivatives hit only the superfield $G$ but not $N_{\alpha \beta}$ because of the restrictions (2.20). Doing so, one can accumulate the factor $W^{2} \bar{W}^{2}$ in the nominator resulting in $\Psi^{2}$ according to (2.39) while the remaining factors can be represented by some function $\tilde{\mathcal{U}}_{2}\left(\Omega^{2}\right)$.

These considerations show that in the long-wave approximation the superconformal action (2.38) simplifies

$$
S_{1}=\int d^{3} x d^{4} \theta G \Psi^{2} \mathcal{H}\left(\Omega^{2}\right)
$$

such that it is described by a single function $\mathcal{H}\left(\Omega^{2}\right)$ of one real variable. There are no any more constraints on the form of this function. This function will be computed explicitly in the next subsection, but in general, it is represented by a power series $\mathcal{H}\left(\Omega^{2}\right)=$ $\sum_{n=0}^{\infty} a_{n} \Omega^{2 n}$ with some coefficients $a_{n}$. The action (2.43) contains the following terms in its component decomposition

$$
S_{1}=\sum_{n=0}^{\infty} a_{n} \int d^{3} x d^{4} \theta \frac{W^{2} \bar{W}^{2}}{2 G^{5}}\left(\frac{1}{8} \frac{N_{\beta}^{\alpha} N_{\alpha}^{\beta}}{G^{4}}\right)^{n}=\sum_{n=0}^{\infty}\left(-\frac{1}{8}\right)^{n+1} a_{n} \int d^{3} x \frac{F^{4+2 n}}{\phi^{5+4 n}}+\ldots
$$


Summing up all together, we conclude that the general form of the superconformal effective action in the long-wave approximation is given by

$$
\Gamma_{\mathcal{N}=2}=\Gamma_{\mathrm{CS}}+\Gamma_{\text {Maxweel }}+\Gamma_{\text {higher }}
$$

where

$$
\begin{aligned}
\Gamma_{\mathrm{CS}} & =c_{0} \int d^{3} x d^{4} \theta V G, \\
\Gamma_{\text {Maxwell }} & =c_{1} \int d^{3} x d^{4} \theta G \ln G, \\
\Gamma_{\text {higher }} & =\int d^{3} x d^{4} \theta G \Psi^{2} \mathcal{H}\left(\Omega^{2}\right) .
\end{aligned}
$$

In components, this action contain the Chern-Simons term (2.21), the Maxwell $F^{2}$ term (2.34) and all higher order terms $F^{2 n}$ with $n \geq 2$ which are written down in (2.44). The undefined coefficients $c_{0}, c_{1}$ and the arbitrary function $\mathcal{H}$ will be found in the next section by explicit quantum computations.

In conclusion of this subsection we comment on the uniqueness of the form of the superconformal action (2.45). The Chern-Simons and the Maxwell terms in this action are fixed by the gauge and superconformal invariance uniquely, up to the coefficients, but the form of the last term $\Gamma_{\text {higher }}$ is not unique. Indeed, we used the ansatz (2.38) which involves only two quasi-primary superfields (2.36), but the other ansätze are also possible. In particular, there is a consequence of descendant quasi-primary superfields for (2.36) given by

$$
\Psi_{n}=\left(\frac{i}{G} \bar{D}^{\alpha} D_{\alpha}\right)^{n} \ln G, \quad \delta \Psi_{n}=\xi \Psi_{n}, \quad n=3,4,5, \ldots,
$$

which can also be used for constructing superconformal invariants in the $\mathcal{N}=2$ superspace,

$$
\tilde{S}=\int d^{3} x d^{4} \theta G \mathcal{A}\left(\Psi, \Omega^{2}, \Psi_{3}, \Psi_{4}, \ldots, \Psi_{n}, \ldots\right), \quad \delta \tilde{S}=0,
$$

where $\mathcal{A}$ is some function. However, these superfields (2.49) are necessary only for describing the terms involving space-time derivatives of the gauge superfields or which are proportional to the free Maxwell equations since in the long-wave approximation (2.20) the action (2.50) can be brought to the form (2.43) by similar manipulations as in (2.42).

\section{Perturbative computations of $\mathcal{N}=2$ and $\mathcal{N}=4$ effective actions}

\subsection{Low-energy effective action for $\mathcal{N}=2$ gauge superfield}

The four-dimensional $\mathcal{N}=1$ and $\mathcal{N}=2$ supersymmetric Euler-Heisenberg effective actions were studied in $[4,10]$ at one loop. The two-loop refining of these results was given 
in [5] and [6] owing to the powerful covariant perturbation theory in superspace elaborated in [16]. Here we apply some of the methods developed in $[4,5,6]$ for studying the structure of the effective action in three-dimensional model of chiral superfield interacting with the background gauge superfield.

The effective action for the model (2.1) can be divided into parity odd and parity even parts,

$$
\Gamma_{\mathcal{N}=2}=\Gamma_{\text {odd }}+\Gamma_{\text {even }}
$$

As soon as the classical action (2.1) is parity even, the appearance of the odd part in the effective action can be only due to the parity anomaly which is studied in details in $[3,17]$ and reviewed in [18]. The anomaly appears owing to the regularization of infrared divergent momentum integrals and yields the term proportional to the Chern-Simons action $\Gamma_{\text {odd }} \propto S_{\mathrm{CS}}$. We will compute $\Gamma_{\text {odd }}$ in the end of this subsection while now we concentrate on $\Gamma_{\text {even }}$.

For $Q$ and $\bar{Q}$ it is convenient to introduce the covariantly (anti)chiral superfields [19],

$$
\mathcal{Q}=Q, \quad \overline{\mathcal{Q}}=\bar{Q} e^{2 V},
$$

which are annihilated by the gauge covariant derivatives (2.16),

$$
\bar{\nabla}_{\alpha} \mathcal{Q}=0, \quad \nabla_{\alpha} \overline{\mathcal{Q}}=0 .
$$

In terms of these superfields the action (2.1) is simply

$$
S_{\mathcal{N}=2}=-\int d^{3} x d^{4} \theta \overline{\mathcal{Q}} \mathcal{Q}
$$

The matrix of second variational derivatives of this action is given by

$$
H=\left(\begin{array}{cc}
\frac{\delta^{2} S}{\delta \mathcal{Q}(z) \delta \mathcal{Q}\left(z^{\prime}\right)} & \frac{\delta^{2} S}{\delta \mathcal{Q}(z) \delta \mathcal{Q}\left(z^{\prime}\right)} \\
\frac{\delta^{2} S}{\delta \mathcal{Q}(z) \delta \mathcal{Q}\left(z^{\prime}\right)} & \frac{\delta^{2} S}{\delta \mathcal{Q}(z) \delta \mathcal{Q}\left(z^{\prime}\right)}
\end{array}\right)=\left(\begin{array}{cc}
0 & \frac{1}{4} \bar{\nabla}^{2} \delta_{-}\left(z, z^{\prime}\right) \\
\frac{1}{4} \nabla^{2} \delta_{+}\left(z, z^{\prime}\right) & 0
\end{array}\right)
$$

where $\delta_{+}$and $\delta_{-}$are covariantly (anti)chiral delta-functions,

$$
\delta_{+}\left(z, z^{\prime}\right)=-\frac{1}{4} \bar{\nabla}^{2} \delta^{7}\left(z-z^{\prime}\right), \quad \delta_{-}\left(z, z^{\prime}\right)=-\frac{1}{4} \nabla^{2} \delta^{7}\left(z-z^{\prime}\right) .
$$

Here $\delta^{7}\left(z-z^{\prime}\right)$ is the delta-function in full $\mathcal{N}=2$ superspace.

The matrix (3.5) leads to the following one-loop effective action,

$$
\Gamma_{\text {even }}=\frac{i}{2} \operatorname{Tr} \ln H=\frac{i}{4} \operatorname{Tr} \ln H^{2}=\frac{i}{4} \operatorname{Tr} \ln \left(\begin{array}{cc}
\frac{1}{16} \bar{\nabla}^{2} \nabla^{2} \delta_{+}\left(z, z^{\prime}\right) & 0 \\
0 & \frac{1}{16} \nabla^{2} \bar{\nabla}^{2} \delta_{-}\left(z, z^{\prime}\right)
\end{array}\right) .
$$

Introducing the covariant (anti)chiral d'Alembertians

$$
\square_{+}=\frac{1}{16} \bar{\nabla}^{2} \nabla^{2}, \quad \square_{-}=\frac{1}{16} \nabla^{2} \bar{\nabla}^{2},
$$


the effective action (3.7) can be rewritten as

$$
\Gamma_{\text {even }}=\frac{i}{4} \operatorname{Tr}_{+} \ln \square_{+}+\frac{i}{4} \operatorname{Tr}_{-} \ln \square_{-},
$$

where $\mathrm{Tr}_{+}$and $\mathrm{Tr}{ }_{-}$denote the functional traces of the corresponding operators in the chiral and antichiral superspaces, respectively. The operators $\square_{+}$and $\square_{-}$acting on the covariantly (anti)chiral superfields have the following representations

$$
\begin{aligned}
& \square_{+}=\nabla^{m} \nabla_{m}+G^{2}+\frac{i}{2}\left(D^{\alpha} W_{\alpha}\right)+i W^{\alpha} \nabla_{\alpha}, \\
& \square_{-}=\nabla^{m} \nabla_{m}+G^{2}-\frac{i}{2}\left(\bar{D}^{\alpha} \bar{W}_{\alpha}\right)-i \bar{W}^{\alpha} \bar{\nabla}_{\alpha} .
\end{aligned}
$$

The terms $D^{\alpha} W_{\alpha}$ and $\bar{D}^{\alpha} \bar{W}_{\alpha}$ in (3.10) and (3.11) can be omitted as soon as we consider the special background, (2.19). Then the operators (3.10) and (3.11) obey the following important properties

$$
\nabla^{2} \square_{+}=\square_{-} \nabla^{2}, \quad \bar{\nabla}^{2} \square_{-}=\square_{+} \bar{\nabla}^{2} .
$$

There are covariantly (anti)chiral Greens functions $G_{+}$and $G_{-}$for these operators,

$$
\square_{+} G_{+}\left(z, z^{\prime}\right)=-\delta_{+}\left(z, z^{\prime}\right), \quad \square_{-} G_{-}\left(z, z^{\prime}\right)=-\delta_{-}\left(z, z^{\prime}\right) .
$$

These Greens functions can be represented by their heat kernels,

$$
G_{ \pm}\left(z, z^{\prime}\right)=i \int_{0}^{\infty} d s K_{ \pm}\left(z, z^{\prime} \mid s\right) e^{-\epsilon s}, \quad \epsilon \rightarrow+0
$$

Further we will omit the factor $e^{-\epsilon s}$ in the integrals over the proper time $s$ for brevity assuming the limit $\epsilon \rightarrow+0$ after calculating the integrals. In terms of the chiral heat kernel the effective action (3.9) reads

$$
\Gamma_{\text {even }}=-\frac{i}{4} \int_{0}^{\infty} \frac{d s}{s} \operatorname{Tr}_{+} K_{+}(s)+c . c .
$$

As a result, the problem of computing the effective action is reduced to finding the coincidence limit of the chiral heat kernel,

$$
\operatorname{Tr}_{+} K_{+}(s)=\int d^{3} x d^{2} \theta K_{+}(z, z \mid s) .
$$

Let us introduce the operator

$$
\square_{\mathrm{v}}=\nabla^{m} \nabla_{m}+G^{2}+i W^{\alpha} \nabla_{\alpha}-i \bar{W}^{\alpha} \bar{\nabla}_{\alpha}
$$

with the Greens function $G_{\mathrm{v}}$ and associated heat kernel $K_{\mathrm{v}}$,

$$
\square_{\mathrm{v}} G_{\mathrm{v}}\left(z, z^{\prime}\right)=-\delta^{7}\left(z, z^{\prime}\right), \quad G_{\mathrm{v}}\left(z, z^{\prime}\right)=i \int_{0}^{\infty} d s K_{\mathrm{v}}\left(z, z^{\prime} \mid s\right) .
$$


For the special background under considerations, $D^{\alpha} W_{\alpha}=\bar{D}^{\alpha} \bar{W}_{\alpha}=0$, this operator has the following important properties

$$
\nabla^{2} \square_{+}=\nabla^{2} \square_{\mathrm{v}}=\square_{\mathrm{v}} \nabla^{2}, \quad \bar{\nabla}^{2} \square_{-}=\bar{\nabla}^{2} \square_{\mathrm{v}}=\square_{\mathrm{v}} \bar{\nabla}^{2},
$$

which are used to relate the (anti)chiral Greens functions (3.13) with $G_{\mathrm{v}}$,

$$
G_{+}\left(z, z^{\prime}\right)=-\frac{1}{4} \bar{\nabla}^{2} G_{\mathrm{v}}\left(z, z^{\prime}\right), \quad G_{-}\left(z, z^{\prime}\right)=-\frac{1}{4} \nabla^{2} G_{\mathrm{v}}\left(z, z^{\prime}\right)
$$

as well as the corresponding heat kernels,

$$
K_{+}\left(z, z^{\prime} \mid s\right)=-\frac{1}{4} \bar{\nabla}^{2} K_{\mathrm{v}}\left(z, z^{\prime} \mid s\right), \quad K_{-}\left(z, z^{\prime} \mid s\right)=-\frac{1}{4} \nabla^{2} K_{\mathrm{v}}\left(z, z^{\prime} \mid s\right) .
$$

Therefore it is sufficient to study the heat kernel $K_{\mathrm{v}}$ while the (anti)chiral ones are deduced from $K_{\mathrm{v}}$ by (3.21).

The heat kernel $K_{\mathrm{v}}$ can be represented as

$$
K_{\mathrm{v}}\left(z, z^{\prime} \mid s\right)=e^{i s\left(\nabla^{m} \nabla_{m}+G^{2}+i W^{\alpha} \nabla_{\alpha}-i \bar{W}^{\alpha} \bar{\nabla}_{\alpha}\right)} \delta^{7}\left(z-z^{\prime}\right) .
$$

For the constant field background $(2.19,2.20)$ there are the following identities

$$
\left[\nabla_{m}, W^{\alpha} \nabla_{\alpha}-\bar{W}^{\alpha} \bar{\nabla}_{\alpha}\right]=0, \quad\left[W^{\alpha} \nabla_{\alpha}-\bar{W}^{\alpha} \bar{\nabla}_{\alpha}, G\right]=0
$$

which allow us to factorize the exponent in (3.22),

$$
K_{\mathrm{v}}\left(z, z^{\prime} \mid s\right)=e^{i s G^{2}} e^{i s\left(i W^{\alpha} \nabla_{\alpha}-i \bar{W}^{\alpha} \bar{\nabla}_{\alpha}\right)} e^{i s\left(\nabla^{m} \nabla_{m}\right)} \delta^{7}\left(z-z^{\prime}\right) \equiv e^{i s G^{2}} \mathcal{O}(s) \tilde{K}\left(z, z^{\prime} \mid s\right),
$$

where

$$
\mathcal{O}(s)=e^{s\left(\bar{W}^{\alpha} \bar{\nabla}_{\alpha}-W^{\alpha} \nabla_{\alpha}\right)}
$$

and the reduced kernel $\tilde{K}\left(z, z^{\prime} \mid s\right)$ solves the equation

$$
\left(i \frac{d}{d s}+\nabla^{m} \nabla_{m}\right) \tilde{K}\left(z, z^{\prime} \mid s\right)=0, \quad \lim _{s \rightarrow 0} \tilde{K}\left(z, z^{\prime} \mid s\right)=\delta^{7}\left(z-z^{\prime}\right) .
$$

Let us consider the following representation for the delta-function in full superspace ${ }^{3}$

$$
\delta^{7}\left(z-z^{\prime}\right)=\int \frac{d^{3} k}{(2 \pi)^{3}} e^{i k^{m} \zeta_{m}} \zeta^{2} \bar{\zeta}^{2}
$$

where $\zeta^{A}$ is $\mathcal{N}=2$ supersymmetric interval,

$$
\zeta^{A}=\left\{\begin{aligned}
\zeta^{\alpha \beta} & =\left(x-x^{\prime}\right)^{\alpha \beta}-2 i\left(\theta-\theta^{\prime}\right)^{(\alpha} \bar{\theta}^{\prime \beta)}+2 i \theta^{\prime(\alpha}\left(\bar{\theta}-\bar{\theta}^{\prime}\right)^{\beta)} \\
\zeta^{\alpha} & =\left(\theta-\theta^{\prime}\right)^{\alpha} \\
\bar{\zeta}^{\alpha} & =\left(\bar{\theta}-\bar{\theta}^{\prime}\right)^{\alpha}
\end{aligned}\right.
$$

\footnotetext{
${ }^{3}$ More generally, one has to insert a parallel displacement operator on the right in (3.27) as well as to further expressions for heat kernels to provide their gauge covariance [16]. However, this is not necessary for the one-loop computations since in the limit of coincident points the covariant derivatives of the parallel displacement operator vanish, $[5,6,16]$.
} 
Using (3.26) and (3.27) we arrive at the following representation for the heat kernel $\tilde{K}$,

$$
\tilde{K}\left(z, z^{\prime} \mid s\right)=\int \frac{d^{3} k}{(2 \pi)^{3}} e^{i k^{n} \zeta_{n}} e^{i s\left(\nabla^{m}+i k^{m}\right)\left(\nabla_{m}+i k_{m}\right)} \zeta^{2} \bar{\zeta}^{2} .
$$

The integration over $d^{3} k$ in (3.29) can be explicitly done, see [16] for the details of similar computations in four-dimensional case,

$$
\tilde{K}\left(z, z^{\prime} \mid s\right)=\frac{1}{8(i \pi s)^{3 / 2}} \sqrt{\operatorname{det}\left(\frac{2 s \mathbf{F}}{1-e^{-2 s \mathbf{F}}}\right)} e^{\frac{i}{4}(\mathbf{F} \operatorname{coth} s \mathbf{F})_{m n} \zeta^{m} \zeta^{n} \zeta^{2} \bar{\zeta}^{2}}
$$

The determinant in (3.30) is over the Lorentz indices of the matrix $\mathbf{F}_{m}{ }^{n}$ introduced in (2.8). This determinant can be explicitly evaluated (see [3] for analogous computations in the non-supersymmetric three-dimensional electrodynamics)

$$
\sqrt{\operatorname{det}\left(\frac{2 s \mathbf{F}}{1-e^{-2 s \mathbf{F}}}\right)}=\frac{s B}{\sinh (s B)},
$$

where

$$
B^{2}=\frac{1}{2} N_{\alpha}^{\beta} N_{\beta}^{\alpha}, \quad N_{\alpha \beta}=D_{(\alpha} W_{\beta)}, \quad \bar{N}_{\alpha \beta}=\bar{D}_{(\alpha} \bar{W}_{\beta)} .
$$

Here the identity (2.14) has been used.

Now we return to the computation of the heat kernel $K_{\mathrm{v}}$ which is expressed in terms of $\tilde{K}$ as in (3.24). For this purpose we need to push the operator $\mathcal{O}(s)$ through the components of the superinterval $\zeta^{A}$ in (3.30). Using the identities

$$
\begin{aligned}
W^{\alpha}(s) & \equiv \mathcal{O}(s) W^{\alpha} \mathcal{O}(-s)=W^{\beta}\left(e^{-s N}\right)_{\beta}{ }^{\alpha} \\
\zeta^{\alpha}(s) & \equiv \mathcal{O}(s) \zeta^{\alpha} \mathcal{O}(-s)=\zeta^{\alpha}+W^{\beta}\left(\left(e^{-s N}-1\right) N^{-1}\right)_{\beta}{ }^{\alpha} \\
\zeta_{\alpha \beta}(s) & \equiv \mathcal{O}(s) \zeta_{\alpha \beta} \mathcal{O}(-s)=\zeta_{\alpha \beta}-2 i \int_{0}^{s} d t\left(W_{(\alpha}(t) \bar{\zeta}_{\beta)}(t)+\bar{W}_{(\alpha}(t) \zeta_{\beta)}(t)\right),
\end{aligned}
$$

we arrive at the following final expression for $K_{\mathrm{v}}$

$$
K_{\mathrm{v}}\left(z, z^{\prime} \mid s\right)=\frac{1}{8(i \pi s)^{3 / 2}} \frac{s B}{\sinh (s B)} e^{i s G^{2}} e^{\frac{i}{4}(\mathbf{F} \operatorname{coth} s \mathbf{F})_{m n} \zeta^{m}(s) \zeta^{n}(s)} \zeta^{2}(s) \bar{\zeta}^{2}(s)
$$

Recall that we need the chiral heat kernel $K_{+}$for the effective action (3.15), which is related to $K_{\mathrm{v}}$ by (3.21). At coincident points, $z=z^{\prime}$, it is easy to argue that the operator $\bar{\nabla}^{2}$ in $(3.21)$ hits only $\bar{\zeta}^{2}(s)$,

$$
-\frac{1}{4} \bar{\nabla}^{2} \bar{\zeta}^{2}(s)=1
$$

Finally, using the identity

$$
\left.\zeta^{2}(s)\right|_{z=z^{\prime}}=s^{2} W^{2} \frac{\sinh ^{2} \frac{s B}{2}}{(s B / 2)^{2}}
$$


we get

$$
K_{+}(s)=K_{+}(z, z \mid s)=\frac{1}{8(i \pi s)^{3 / 2}} s^{2} W^{2} e^{i s G^{2}} \frac{\tanh (s B / 2)}{s B / 2} .
$$

The corresponding one-loop effective action (3.15) reads

$$
\Gamma_{\text {even }}=-\frac{1}{32 \pi} \int d^{3} x d^{2} \theta \int_{0}^{\infty} \frac{d s}{\sqrt{i \pi s}} W^{2} e^{i s G^{2}} \frac{\tanh (s B / 2)}{s B / 2}+\text { c.c. },
$$

where $B$ is given by (3.32), $B^{2}=\frac{1}{2} D_{\alpha} W^{\beta} D_{\beta} W^{\alpha}$. Finally, we rewrite (3.40) in the full $\mathcal{N}=2$ superspace,

$$
\Gamma_{\text {even }}=\frac{1}{4 \pi} \int d^{3} x d^{4} \theta\left[G \ln G+\frac{1}{4} \int_{0}^{\infty} \frac{d s}{\sqrt{i \pi s}} e^{i s G^{2}} \frac{W^{2} \bar{W}^{2}}{B^{2}}\left(\frac{\tanh (s B / 2)}{s B / 2}-1\right)\right] .
$$

The superfield strength $G$ in (3.40) serves as an effective massive regularizator for infrared divergencies. In fact, giving a non-zero vev $\langle G\rangle \neq 0$ generates a mass for the matter superfield which is equal to the central charge of the $\mathcal{N}=2$ superalgebra. In other words, we derived the effective action (3.40) in the Coulomb branch of the $\mathcal{N}=2$ supergauge theory. Alternatively, one can consider standard mass term $m \int d^{3} x d^{2} \theta Q^{2}$, but it violates the parity and requires more accurate considerations. These issues were studied in details in [20]. We will consider the hypermultiplet model with the complex mass in the next section.

Now we come back to the derivation of the parity odd part of the effective action (3.1). The reason why both $\Gamma_{\text {odd }}$ and $\Gamma_{\text {even }}$ cannot be derived in the unified procedure given above is quite similar to the non-supersymmetric case considered in [3]: The ChernSimons term formally vanishes in the approximation of the constant fields (2.20), but the variation of the Chern-Simons term with respect to the gauge superfield produces a non-vanishing current. Therefore the Chern-Simons term in the effective action can be obtained by integrating the variation

$$
\delta \Gamma_{\mathcal{N}=2}=\int d^{3} x d^{4} \theta \delta V\langle J\rangle
$$

where $\langle J\rangle$ is the effective current,

$$
\langle J\rangle=\left\langle\frac{\delta S}{\delta V}\right\rangle=-2\langle\overline{\mathcal{Q}} \mathcal{Q}\rangle .
$$

The propagator $\langle\overline{\mathcal{Q}} \mathcal{Q}\rangle$ is expressed in terms of the Greens function (3.18),

$$
i\left\langle\overline{\mathcal{Q}}(z) \mathcal{Q}\left(z^{\prime}\right)\right\rangle=\frac{1}{16} \bar{\nabla}^{2} \nabla^{\prime 2} G_{\mathrm{v}}\left(z, z^{\prime}\right) .
$$

Using the explicit form (3.36) for $G_{\mathrm{v}}$, we find

$$
\langle J\rangle=\left.\frac{i}{8} \bar{\nabla}^{2} \nabla^{\prime 2} G_{\mathrm{v}}\left(z, z^{\prime}\right)\right|_{z=z^{\prime}}=-\frac{1}{4} \int_{0}^{\infty} \frac{d s}{(i \pi s)^{3 / 2}} \frac{s B}{\sinh (s B)} e^{i s G^{2}} .
$$


The effective current (3.45) contains both finite and infrared divergent parts. All finite contributions to the effective action are parity even and are already taken into account in (3.40). The parity odd contributions arise from the divergent part which reads

$$
\langle J\rangle_{\operatorname{div}}=-\frac{1}{4} \int_{0}^{\infty} \frac{d s}{(i \pi s)^{3 / 2}} e^{i s G^{2}}
$$

Regularizing this integral appropriately we find

$$
\langle J\rangle_{\mathrm{reg}}=\frac{G}{2 \pi} .
$$

Substituting this current into (3.42) we obtain the odd part of the effective action,

$$
\Gamma_{\text {odd }}=\frac{1}{4 \pi} \int d^{3} x d^{4} \theta V G .
$$

Summing up (3.40) with (3.48) we get the resulting effective action in the form (2.45) with

$$
\begin{aligned}
\Gamma_{\mathrm{CS}} & =\frac{1}{4 \pi} \int d^{3} x d^{4} \theta V G \\
\Gamma_{\text {Maxwell }} & =\frac{1}{4 \pi} \int d^{3} x d^{4} \theta G \ln G \\
\Gamma_{\text {higher }} & =\frac{1}{32 \pi} \int d^{3} x d^{4} \theta G \frac{\Psi^{2}}{\Omega^{2}} \int_{0}^{\infty} \frac{d t e^{i t}}{\sqrt{i \pi t}}\left(\frac{\tanh (t \Omega)}{t \Omega}-1\right) .
\end{aligned}
$$

The action (3.51) is obtained from (3.41) by changing to the dimensionless integration variable $t=s G^{2}$ and then by rewriting it in terms of the quasi-primary superconformal superfields $(2.36)$ with the help of $(2.39,2.40)$. As is demonstrated in the previous section each of the actions (3.49), (3.50) and (3.51) is explicitly superconformal.

Representing the effective action (3.41) in the superconformal form $(3.50,3.51)$ allows us to relax the on-shell constraint (2.19). Indeed, there are infinitely many ways of complementing the effective action (3.41) by the terms vanishing on the classical equations of motion, but the superconformal invariance fixes this freedom and gives the unique answer $(3.50,3.51)$ for such an action. Therefore we conclude that $(3.49,3.50,3.51)$ are correct offshell contributions to the low-energy effective action of the chiral superfield interacting with the background gauge superfield. These conclusions are completely analogous to the ones in [10] for the four-dimensional $\mathcal{N}=2$ superconformal theories.

In principle, one can think that the superconformal invariance allows one to go beyond the long-wave approximation (2.20), but it is not completely true because when the space-time derivatives are taken into account the terms (3.50) and (3.51) in the effective action may be corrected by some contributions involving the higher-order superconformal invariants (2.50). The analysis of contributions to the effective action with space-time derivatives is a hard task and therefore we restrict ourself to the long-wave approximation $(2.20)$. 
An interesting feature of the three-dimensional theory is that the proper time integral in (3.40) can be expressed in terms of special functions. In particular, for real $B$ (constant electric field) this integral is represented by the following combination of generalized Riemann zeta functions, ${ }^{4}$

$$
\Gamma_{\text {even }}=\frac{1-i}{4 \pi} \int d^{3} x d^{4} \theta \frac{W^{2} \bar{W}^{2}}{B^{5 / 2}}\left[\zeta\left(-\frac{1}{2},-\frac{i G^{2}}{2 B}\right)-2 \zeta\left(-\frac{1}{2}, \frac{1}{2}-\frac{i G^{2}}{2 B}\right)+\zeta\left(-\frac{1}{2}, 1-\frac{i G^{2}}{2 B}\right)\right] .
$$

This representation allows us to consider strong electric field background, $B \gg 1$,

$$
\Gamma_{\text {even }}=\frac{1-i}{4 \pi} \int d^{3} x d^{4} \theta \frac{W^{2} \bar{W}^{2}}{B^{5 / 2}}\left[(\sqrt{2}-4) \zeta(-1 / 2)+O\left(B^{-1}\right)\right] .
$$

The non-vanishing imaginary part of the effective action shows the vacuum instability for strong electric field. For imaginary $B$ (constant magnetic field) one can replace $B \rightarrow-i B$ in (3.52) to see that the effective action is real for any value of the field.

\subsection{Low-energy effective action for $\mathcal{N}=4$ gauge multiplet}

The classical action for $\mathcal{N}=4, d=3$ hypermultiplet appears by dimensional reduction from $\mathcal{N}=2, d=4$ hypermultiplet which is described in $\mathcal{N}=1, d=4$ superspace in $[12,19]$. In our case it is given by a pair of chiral superfields $\left(Q_{+}, Q_{-}\right)$in the $\mathcal{N}=2$, $d=3$ superspace where the subscripts ' + ' and ' - ' stress that these superfields have corresponding charges with respect to the gauge superfield. We consider the minimal gauge interaction of the hypermultiplet with the $\mathcal{N}=4$ vector multiplet described by the pair $(V, \Phi)$, where $V$ is a real gauge $\mathcal{N}=2$ superfield and $\Phi$ is a chiral $\mathcal{N}=2$ superfield. The corresponding massless action reads

$$
S_{\mathcal{N}=4}=-\int d^{3} x d^{4} \theta\left(\bar{Q}_{+} e^{2 V} Q_{+}+\bar{Q}_{-} e^{-2 V} Q_{-}\right)-\left(\int d^{3} x d^{2} \theta Q_{+} \Phi Q_{-}+\text {c.c. }\right) .
$$

The massive case can be obtained from (3.54) by the shift $\Phi \rightarrow \Phi+m$ with $m$ being a complex mass parameter.

It is convenient to unify the chiral superfields $Q_{+}$and $Q_{-}$with opposite charges to a chiral doublet $\mathbf{Q}[5]$,

$$
\mathbf{Q}=\exp \left(i \frac{\pi}{4} \sigma_{1}\right)\left(\begin{array}{c}
Q_{+} \\
Q_{-}
\end{array}\right)
$$

while for the gauge superfield $V$ and its superfield strengths we introduce

$$
\mathbf{V}=\sigma_{2} V, \quad \mathbf{G}=\sigma_{2} G, \quad \mathbf{W}_{\alpha}=\sigma_{2} W_{\alpha}, \quad \overline{\mathbf{W}}_{\alpha}=\sigma_{2} \bar{W}_{\alpha}
$$

\footnotetext{
${ }^{4}$ There is a definition of the generalized Riemann zeta function, $\zeta(s, q)=\sum_{n=0}^{\infty}(q+n)^{-s}$, valid for $\operatorname{Re}(s)>1, \operatorname{Re}(q)>0$, but it can be analytically continued for other values of arguments. This function is also referred to as Hurwitz zeta function (see, e.g., [21]).
} 
where $\sigma_{1}, \sigma_{2}, \sigma_{3}$ are the Pauli matrices. Then the action (3.54) reads

$$
S_{\mathcal{N}=4}=-\int d^{3} x d^{4} \theta \overline{\mathbf{Q}}^{\mathrm{T}} e^{2 \mathbf{V}} \mathbf{Q}+\left(\frac{i}{2} \int d^{3} x d^{2} \theta \Phi \mathbf{Q}^{\mathrm{T}} \mathbf{Q}+\text { c.c. }\right) .
$$

We will also use the covariant spinor derivatives covariantized by the matrix gauge superfield (3.56),

$$
\nabla_{\alpha}=D_{\alpha}+2 D_{\alpha} \mathbf{V}, \quad \bar{\nabla}_{\alpha}=\bar{D}_{\alpha},
$$

as well as the covariantly chiral superfields,

$$
\overline{\mathcal{Q}}=e^{-2 \mathbf{V}} \overline{\mathbf{Q}}, \quad \mathcal{Q}=\mathbf{Q} .
$$

With these notations the action (3.57) takes the form

$$
S_{\mathcal{N}=4}=-\int d^{3} x d^{4} \theta \overline{\mathcal{Q}}^{\mathrm{T}} \mathcal{Q}+\left(\frac{i}{2} \int d^{3} x d^{2} \theta \Phi \mathcal{Q}^{\mathrm{T}} \mathcal{Q}+\text { c.c. }\right) .
$$

We are interested in the one-loop effective action $\Gamma_{\mathcal{N}=4}[V, \Phi]$ in the model $(3.54)$ which is obtained by integrating out the charged hypermultiplet with $V$ and $\Phi$ being the background superfields. The constraints on the considered vector background $(2.19,2.20)$ should be extended by the following constraint on $\Phi$,

$$
D_{\alpha} \Phi=0 \text {. }
$$

Similarly as in the previous subsection, we compute the matrix of second variational derivatives,

$$
H=\left(\begin{array}{cc}
\frac{\delta^{2} S_{\mathcal{N}=4}}{\delta \mathcal{Q}(z) \delta \mathcal{Q}\left(z^{\prime}\right)} & \frac{\delta^{2} S_{\mathcal{N}=4}}{\delta \mathcal{Q}(z) \delta \mathcal{Q}\left(z^{\prime}\right)} \\
\frac{\delta^{2} S_{\mathcal{N}=4}}{\delta \mathcal{Q}(z) \delta \mathcal{Q}\left(z^{\prime}\right)} & \frac{\delta^{2} S_{\mathcal{N}=4}}{\delta \overline{\mathcal{Q}}(z) \delta \overline{\mathcal{Q}}\left(z^{\prime}\right)}
\end{array}\right)=\left(\begin{array}{cc}
i \Phi \delta_{+}\left(z, z^{\prime}\right) & \frac{1}{4} \bar{\nabla}^{2} \delta_{-}\left(z, z^{\prime}\right) \\
\frac{1}{4} \nabla^{2} \delta_{+}\left(z, z^{\prime}\right) & i \bar{\Phi} \delta_{-}\left(z, z^{\prime}\right)
\end{array}\right)
$$

where $\delta_{+}$and $\delta_{-}$are gauge covariant (anti)chiral delta-functions defined with respect to the gauge covariant derivatives (3.58). Then the one-loop effective action reads [5]

$$
\Gamma_{\mathcal{N}=4}=\frac{i}{2} \operatorname{Tr} \ln H=\frac{i}{2} \operatorname{Tr}+\ln \left(\square_{+}+\frac{1}{16} \bar{\nabla}^{2} \bar{\Phi} \frac{1}{\square_{-}} \nabla^{2} \Phi\right)+\text { c.c. }
$$

Here $\operatorname{Tr}$ takes into account not only the functional trace of the corresponding operators, but also the matrix trace since we deal with the matrix gauge superfield (3.56). One can easily see that the matrix trace gives extra coefficient 2 in (3.63) as compared to (3.9).

For the considered background (3.61) the expression (3.63) simplifies,

$$
\Gamma_{\mathcal{N}=4}=\frac{i}{2} \operatorname{Tr}_{+} \ln \left(\square_{+}+\bar{\Phi} \Phi\right)+\text { c.c. }
$$

Hence, we can immediately write down the answer for the effective action (3.64) by making the R-invariant shift $G^{2} \rightarrow G^{2}+\bar{\Phi} \Phi$ in the action (3.40),

$$
\Gamma_{\mathcal{N}=4}=-\frac{1}{16 \pi} \int d^{3} x d^{2} \theta \int_{0}^{\infty} \frac{d s}{\sqrt{i \pi s}} W^{2} e^{i s\left(G^{2}+\bar{\Phi} \Phi\right)} \frac{\tanh (s B / 2)}{s B / 2}+\text { c.c. },
$$


or, in full $\mathcal{N}=2$ superspace it reads

$$
\begin{aligned}
\Gamma_{\mathcal{N}=4}= & \frac{1}{2 \pi} \int d^{3} x d^{4} \theta\left[-\sqrt{G^{2}+\bar{\Phi} \Phi}+G \ln \left(G+\sqrt{G^{2}+\bar{\Phi} \Phi}\right)\right. \\
& \left.+\frac{1}{4} \int_{0}^{\infty} \frac{d s}{\sqrt{i \pi s}} e^{i s\left(G^{2}+\bar{\Phi} \Phi\right)} \frac{W^{2} \bar{W}^{2}}{B^{2}}\left(\frac{\tanh (s B / 2)}{s B / 2}-1\right)\right] .
\end{aligned}
$$

We point out that there is no Chern-Simons term induced by the quantum corrections from $\mathcal{N}=4$ hypermultiplet as soon as this model has no parity anomaly, see, e.g., [18] for a review. This was also checked in [22] by explicit quantum computations in $\mathcal{N}=3$, $d=3$ harmonic superspace.

Similarly as in the $\mathcal{N}=2$ case, the effective action (3.66) should be superconformal. The terms in the first line of (3.66) obviously respect the $\mathcal{N}=2$ superconformal symmetry because the superfield $\bar{\Phi} \Phi$ transforms under the superconformal group in the same way as $G^{2}$. However, the second line of (3.66) needs to be rewritten in a superconformal form. For this purpose we consider the following generalizations of the superconformal quasi-primary superfields (2.36)

$$
\begin{aligned}
\boldsymbol{\Psi} & =\frac{i}{G} \bar{D}^{\alpha} D_{\alpha} \ln \left(G+\sqrt{G^{2}+\bar{\Phi} \Phi}\right) \\
\boldsymbol{\Omega}^{2} & =\frac{1}{8} \frac{1}{\sqrt{G^{2}+\bar{\Phi} \Phi}} \bar{D}^{\alpha} D_{\alpha} \frac{1}{G} \bar{D}^{\beta} D_{\beta} \ln \left(G+\sqrt{G^{2}+\bar{\Phi} \Phi}\right) .
\end{aligned}
$$

It is easy to see that these superfields are $\mathcal{N}=2$ quasi-primary and transform as in (2.37). When the gauge multiplet is constrained by $(2.20,2.19,3.61)$, the superfields $(3.67)$ can be represented as follows

$$
\begin{aligned}
\Psi & =-i \frac{\bar{W}^{\alpha} W_{\alpha}}{\left(G^{2}+\bar{\Phi} \Phi\right)^{3 / 2}}, \\
\boldsymbol{\Omega}^{2} & =\frac{1}{8} \frac{N_{\beta}^{\alpha} N_{\alpha}^{\beta}}{\left(G^{2}+\bar{\Phi} \Phi\right)^{2}}+\frac{3}{4} \frac{G N^{\alpha \beta} W_{\alpha} \bar{W}_{\beta}}{\left(G^{2}+\bar{\Phi} \Phi\right)^{3}}+\frac{15}{16} \frac{G^{2} W^{2} \bar{W}^{2}}{\left(G^{2}+\bar{\Phi} \Phi\right)^{4}} .
\end{aligned}
$$

These representations allow us to rewrite the effective action (3.66) in the $\mathcal{N}=2$ superconformal form,

$$
\begin{aligned}
\Gamma_{\mathcal{N}=4}= & \frac{1}{2 \pi} \int d^{3} x d^{4} \theta\left[-\sqrt{G^{2}+\bar{\Phi} \Phi}+G \ln \left(G+\sqrt{G^{2}+\bar{\Phi} \Phi}\right)\right. \\
& \left.+\frac{1}{8} \frac{\Psi^{2}}{\Omega^{2}} \sqrt{G^{2}+\bar{\Phi} \Phi} \int_{0}^{\infty} \frac{d t e^{i t}}{\sqrt{i \pi t}}\left(\frac{\tanh (t \Omega)}{t \Omega}-1\right)\right]
\end{aligned}
$$

Now we relax the constraint (2.19) and conclude that (3.69) is the off-shell low-energy effective action for the $\mathcal{N}=4$ gauge multiplet in the long-wave approximation.

Let us single out the terms in the first line in (3.69),

$$
\int d^{3} x d^{4} \theta\left[-\sqrt{G^{2}+\bar{\Phi} \Phi}+G \ln \left(G+\sqrt{G^{2}+\bar{\Phi} \Phi}\right)\right] .
$$


It is easy to see that (3.70) can be obtained by the dimensional reduction from the action of $\mathcal{N}=2, d=4$ improved tensor multiplet formulated in the $\mathcal{N}=1, d=4$ superspace in [23] which was recently revisited in [24]. In the three-dimensional case the action of the form (3.70) was studied in [7]. ${ }^{5}$

It is interesting to note that (3.70) was recently obtained in [25] as a dual representation of the classical action of the Abelian Gaiotto-Witten model. The Gaiotto-Witten model [26] is the $\mathcal{N}=4$ supersymmetric Chern-Simons-matter theory with one hypermultiplet in the bifundamental representation of the twisted gauge group $\mathcal{G}_{1} \times \mathcal{G}_{2}$ where the gauge superfields corresponding to these two groups $\mathcal{G}_{1}$ and $\mathcal{G}_{2}$ have Chern-Simons rather SYM kinetic terms. The authors of [25] showed that in the Abelian case one of these gauge superfields together with the hypermultiplet can be eliminated from the classical action resulting in the action for the second gauge superfield which appeared to have the form (3.70). Hence, the classical action of the Abelian Gaiotto-Witten model in the representation (3.70) arises as the leading term in the effective action in the charged hypermultiplet model.

Our final comment is that the effective action (3.70) hints the form of the effective Kähler superpotential. Indeed, for the vanishing gauge superfield, $G=0$, the expression (3.70) reduces to

$$
\int d^{3} x d^{4} \theta \sqrt{\bar{\Phi} \Phi} \propto \int d^{3} x \frac{1}{\sqrt{\bar{\varphi} \varphi}} \partial^{m} \bar{\varphi} \partial_{m} \varphi+\ldots
$$

where $\varphi$ is the lowest component of $\Phi$ and dots stand for the terms involving other component fields. It would be interesting to do an independent computation of the Kähler superpotential as a part of the effective action in the three-dimensional $\mathcal{N}=2$ WessZumino model.

\section{Summary and discussion}

In this paper we studied the one-loop effective action for three-dimensional $\mathcal{N}=2$ and $\mathcal{N}=4$ gauge superfields induced by quantum supersymmetric matter fields. We restrict ourself to the long-wave approximation when the background gauge superfield is constant with respect to the space-time coordinates and obeys the free supersymmetric Maxwell equations. In the non-supersymmetric case such an action is known as the EulerHeisenberg effective action which was studied for the three-dimensional electrodynamics in [3]. The present work is a supersymmetric generalization of the results of [3].

Before computing the effective action in the model of the $\mathcal{N}=2$ chiral superfield interacting with the background gauge superfield we found a general form (2.45) of such an action subject to the constraints of the gauge and superconformal invariance. The leading terms in this action are given by the Chern-Simons term (2.46) and by a superconformal generalization of the Maxwell action (2.47). The functional form of these two terms is fixed by the superconformal invariance uniquely, up to the coefficients. The higher order terms with respect to the Maxwell field strength are taken into account by the action

\footnotetext{
${ }^{5}$ We are grateful to S.M. Kuzenko for drawing our attention to the papers $[7,23,24]$.
} 
(2.48) which is found up to one arbitrary function $\mathcal{H}$ of quasi-primary superfields (2.36) in the $\mathcal{N}=2$ superspace which are constructed in terms of the superfield strength $G$ and its covariant spinor derivatives. This analysis is quite similar to [10] where the lowenergy effective action in the $\mathcal{N}=2, d=4$ supergauge theory was expressed in terms of superconformal invariants.

After considering the general structure of the superconformal action we explicitly compute it by integrating out the chiral superfields interacting with the background gauge superfield. The results of the calculations match the previously proposed form (2.45): The coefficients in Chern-Simons and Maxwell terms are fixed as in (3.49) and (3.50) while the higher-order contributions with respect to the Maxwell field strengths are represented by the action (3.51) which is expressed in terms of the quasi-primary superfields (2.36).

The effective action for the $\mathcal{N}=4$ gauge superfield is obtained in the form (3.66). It has no Chern-Simons term since there is no parity anomaly for the model of charged hypermultiplet (see, e.g., [18] for a review). The absence of the Chern-Simons term in the charged hypermultiplet was also checked in our recent work [22] using direct quantum computations in the $\mathcal{N}=3, d=3$ harmonic superspace. Therefore the effective action (3.66) starts from the Maxwell term (written in the $\mathcal{N}=2$ superspace in a superconformal form) as well as contains all higher orders of the Maxwell field strength in components. It is interesting to note that the leading terms without derivatives in the effective action for the $\mathcal{N}=4$ gauge superfield coincide with the classical action of the Abelian GaiottoWitten model rewritten in [25] in terms of dynamical gauge superfield. Therefore one can consider the Abelian Gaiotto-Witten model as the effective theory induced by quantum hypermultiplet superfield.

One of the applications of the obtained effective actions for the $\mathcal{N}=2$ and $\mathcal{N}=4$ gauge theories may be given within the study of the mirror symmetry [27] for three-dimensional gauge theories. The mirror symmetry is a kind of dualities for three-dimensional gauge theories which relates one field theory at strong coupling with another theory in the perturbative regime. In particular, the leading term (3.50) in the $\mathcal{N}=2$ gauge superfield effective action is known to be dual to the Kähler sigma model which was studied in [28]. As soon as we derived not only the leading term (3.50), but also a number of derivative contributions (3.51) in the $\mathcal{N}=2$ effective action, it is natural to find the corrections to the sigma model considered in [28] due to the terms (3.51). In a similar way it would be interesting to explore the duality for the $\mathcal{N}=4$ gauge superfield effective action (3.69). Note that modern applications of the mirror symmetry for three-dimensional models with $\mathcal{N}=2$ and $\mathcal{N}=4$ supersymmetry are helpful for the studies of the ABJM-like theories $[29]$.

It is natural to consider the $\mathcal{N}=2$ chiral superfield interacting with the background gauge superfield and the $\mathcal{N}=4$ charged hypermultiplet as the parts of the $\mathcal{N}=2$ and $\mathcal{N}=4$ supersymmetric three-dimensional electrodynamics, respectively. In this case the one-loop Euler-Heisenberg-type effective actions obtained in the present paper receive two-loop (as well as all higher-loop) corrections which are tempting to study. For the four-dimensional supersymmetric electrodynamics the two-loop corrections to the supersymmetric Euler-Heisenberg effective action were computed in $[5,6]$, but in the 
three-dimensional case this problem has never been addressed. Finally, it is interesting to study the effective action in the non-Abelian $\mathcal{N}=2$ and $\mathcal{N}=4$ three-dimensional supergauge models and then to extend these results to the theories with $\mathcal{N}=6$ and $\mathcal{N}=8$ supersymmetry which are worldvolume field theories of M2 and D2 branes. It would open the possibility to study the effective actions in the BLG and ABJM theories which would give an effective quantum description of multiple M2 branes. There are also various deformations of the BLG and ABJM models [30, 31] which are interesting from the point of view of the $\mathrm{AdS}_{4} / \mathrm{CFT}_{3}$ correspondence because they correspond to the infrared stable superconformal points in the three-dimensional $\mathcal{N}=2$ supergauge theories $[31,32,33]$. It is natural to study the problem of effective action in these models as well.

\section{Acknowledgments}

We are grateful to S.M. Kuzenko for useful comments. I.B.S. is indebted to O. Lechtenfeld for helpful discussions and to ITP, Leibniz Universität Hannover for kind hospitality where

a part of this work was done. The present work is supported by RFBR grant, project No 09-02-00078 and by a grant for LRSS, project No 3558.2010.2. I.L.B. and I.B.S. acknowledge the support from the RFBR grants No 10-02-90446 and No 09-02-91349 as well as from a DFG grant, project No 436 RUS/113/669. The work of I.B.S. is also supported by the fellowship of the Dynasty foundation. N.G.P. acknowledges the support from RFBR grant, project No 08-02-00334.

\section{Appendices}

\section{A. $\mathcal{N}=2$ superspace conventions}

In the present paper we use the conventions for the three-dimensional gamma matrices following our previous works $[22,34]$. In particular, the gamma matrices $\left(\gamma^{0}\right)_{\alpha}^{\beta}=-i \sigma_{2}$, $\left(\gamma^{1}\right)_{\alpha}^{\beta}=\sigma_{3},\left(\gamma^{2}\right)_{\alpha}^{\beta}=\sigma_{1}$ obey the Clifford algebra

$$
\left\{\gamma^{m}, \gamma^{n}\right\}=-2 \eta^{m n}, \quad \eta^{m n}=\operatorname{diag}(1,-1,-1),
$$

and the following orthogonality and completeness relations

$$
\left(\gamma^{m}\right)_{\alpha \beta}\left(\gamma^{n}\right)^{\alpha \beta}=2 \eta^{m n}, \quad\left(\gamma^{m}\right)_{\alpha \beta}\left(\gamma_{m}\right)^{\rho \sigma}=\left(\delta_{\alpha}^{\rho} \delta_{\beta}^{\sigma}+\delta_{\alpha}^{\sigma} \delta_{\beta}^{\rho}\right)
$$

We raise and lower the spinor indices with the $\varepsilon$-tensor, e.g., $\left(\gamma_{m}\right)_{\alpha \beta}=\varepsilon_{\alpha \sigma}\left(\gamma_{m}\right)_{\beta}^{\sigma}, \varepsilon_{12}=1$. Any vector index can be converted into a pair of spinor ones by the following rules

$$
\begin{aligned}
x^{\alpha \beta} & =\left(\gamma_{m}\right)^{\alpha \beta} x^{m}, & x^{m} & =\frac{1}{2}\left(\gamma^{m}\right)_{\alpha \beta} x^{\alpha \beta}, \\
\partial_{\alpha \beta} & =\left(\gamma^{m}\right)_{\alpha \beta} \partial_{m}, & \partial_{m} & =\frac{1}{2}\left(\gamma_{m}\right)^{\alpha \beta} \partial_{\alpha \beta},
\end{aligned}
$$

so that

$$
\partial_{m} x^{n}=\delta_{m}^{n}, \quad \partial_{\alpha \beta} x^{\rho \sigma}=\delta_{\alpha}^{\rho} \delta_{\beta}^{\sigma}+\delta_{\alpha}^{\sigma} \delta_{\beta}^{\rho}=2 \delta_{\alpha}^{(\rho} \delta_{\beta}^{\sigma)}
$$


The $\mathcal{N}=2, d=3$ superspace is parametrized by the coordinates $z^{M}=\left(x^{m}, \theta_{\alpha}, \bar{\theta}_{\alpha}\right)$ with $\bar{\theta}_{\alpha}=\left(\theta_{\alpha}\right)^{*}$. The covariant spinor derivatives

$$
D_{\alpha}=\frac{\partial}{\partial \theta^{\alpha}}+i \bar{\theta}^{\beta} \partial_{\alpha \beta}, \quad \bar{D}_{\alpha}=-\frac{\partial}{\partial \bar{\theta}^{\alpha}}-i \theta^{\beta} \partial_{\alpha \beta}
$$

obey the standard anticommutation relation

$$
\left\{D_{\alpha}, \bar{D}_{\beta}\right\}=-2 i \partial_{\alpha \beta} .
$$

The integration measure in the full $\mathcal{N}=2, d=3$ superspace is defined as

$$
d^{7} z \equiv d^{3} x d^{4} \theta=\frac{1}{16} d^{3} x D^{2} \bar{D}^{2}, \quad \text { so that } \int d^{3} x f(x)=\int d^{7} z \theta^{2} \bar{\theta}^{2} f(x),
$$

for some field $f(x)$. Here we use the following conventions for contractions of the spinor indices

$$
D^{2}=D^{\alpha} D_{\alpha}, \quad \bar{D}^{2}=\bar{D}^{\alpha} \bar{D}_{\alpha}, \quad \theta^{2}=\theta^{\alpha} \theta_{\alpha}, \quad \bar{\theta}^{2}=\bar{\theta}^{\alpha} \bar{\theta}_{\alpha} .
$$

The chiral subspace is parametrized by $z_{+}=\left(x_{+}^{m}, \theta_{\alpha}\right)$, where $x_{ \pm}^{m}=x^{m} \pm i \gamma_{\alpha \beta}^{m} \theta^{\alpha} \bar{\theta}^{\beta}$. The chiral superfields are defined as usual, $\bar{D}_{\alpha} \Phi=0 \Rightarrow \Phi=\Phi\left(x_{+}^{m}, \theta_{\alpha}\right)$. The integration

measure in the chiral superspace $d^{5} z \equiv d^{3} x d^{2} \theta$ is related to the full superspace measure (A.7) as

$$
d^{7} z=-\frac{1}{4} d^{5} z \bar{D}^{2}
$$

\section{B. Superconformal transformations in $\mathcal{N}=2$ superspace}

Here we review a representation of the superconformal group on the superfields in the $\mathcal{N}=2, d=3$ superspace which was used in Section 2.2 (see some details in [11], the analogous construction for $\mathcal{N}=1, d=4$ superspace was given in [12]).

Let us consider the infinitesimal superconformal transformations of coordinates of the $\mathcal{N}=2$ superspace $z^{A}=\left(x^{\alpha \beta}, \theta^{\alpha}, \bar{\theta}^{\alpha}\right)$,

$$
z^{A} \longrightarrow z^{A}+\delta_{\mathrm{sc}} z^{A}
$$

where $\delta_{\mathrm{sc}} z^{A}$ explicitly reads

$$
\begin{aligned}
\delta_{\mathrm{sc}} x^{\alpha \beta}= & a x^{\alpha \beta}+x^{\alpha \rho} x^{\beta \gamma} k_{\rho \gamma}-\frac{1}{2} \theta^{2} \bar{\theta}^{2} k^{\alpha \beta}+2 i \theta^{(\alpha} x^{\beta) \gamma} \eta_{\gamma}+\theta^{2} \bar{\theta}^{(\alpha} \eta^{\beta)} \\
& +2 i \bar{\theta}^{(\alpha} x^{\beta) \gamma} \bar{\eta}_{\gamma}+\bar{\theta}^{2} \theta^{(\alpha} \bar{\eta}^{\beta)} \\
\delta_{\mathrm{sc}} \theta^{\alpha}= & (a / 2+i b) \theta^{\alpha}+\theta^{\beta} x^{\alpha \gamma} k_{\beta \gamma}+\frac{i}{2} \theta^{2} \bar{\theta}^{\beta} k_{\beta}^{\alpha}+i \theta^{2} \eta^{\alpha}+\left(x^{\alpha \beta}+2 i \theta^{(\alpha} \bar{\theta}^{\beta)}\right) \bar{\eta}_{\beta}, \\
\delta_{\mathrm{sc}} \bar{\theta}^{\alpha}= & (a / 2-i b) \bar{\theta}^{\alpha}+\bar{\theta}^{\beta} x^{\alpha \gamma} k_{\beta \gamma}+\frac{i}{2} \bar{\theta}^{2} \theta^{\beta} k_{\beta}^{\alpha}+i \bar{\theta}^{2} \bar{\eta}^{\alpha}+\left(x^{\alpha \beta}-2 i \theta^{(\alpha} \bar{\theta}^{\beta)}\right) \eta_{\beta} .
\end{aligned}
$$

Here $a, b, k_{\alpha \beta}, \eta_{\alpha}, \bar{\eta}_{\alpha}$ are the parameters of dilatations, $\mathrm{U}(1)$ transformations, special conformal transformations and S-supersymmetry transformations, respectively. These transformations can be shown to obey the superconformal algebra $\operatorname{osp}(2, \mathbb{R} \mid 2)$. 
Let us consider a superconformal Killing vector $\xi^{A}=\xi^{A}(z)=\left(\xi^{\alpha \beta}(z), \xi^{\alpha}(z), \bar{\xi}^{\alpha}(z)\right)$, where

$$
\xi^{\alpha \beta}=\delta_{\mathrm{sc}} x^{\alpha \beta}-2 i \delta_{\mathrm{sc}} \theta^{(\alpha} \bar{\theta}^{\beta)}+2 i \theta^{(\alpha} \delta_{\mathrm{sc}} \bar{\theta}^{\beta)}, \quad \xi^{\alpha}=\delta_{\mathrm{sc}} \theta^{\alpha}, \quad \bar{\xi}^{\alpha}=\delta_{\mathrm{sc}} \bar{\theta}^{\alpha} .
$$

The explicit expressions for the components of the superconformal Killing vector can be derived from (B.2)-(B.4),

$$
\begin{aligned}
\xi^{\alpha \beta}= & a x^{\alpha \beta}+4 b \theta^{(\alpha} \bar{\theta}^{\beta)}+k_{\gamma \delta} x_{+}^{\gamma(\alpha} x_{-}^{\beta) \delta}-2 i x^{\gamma(\alpha} k_{\gamma}^{\beta)} \theta^{\rho} \bar{\theta}_{\rho} \\
& +4 i \theta^{(\alpha} x_{-}^{\beta) \gamma} \eta_{\gamma}+4 i \bar{\theta}^{(\alpha} x_{+}^{\beta) \gamma} \bar{\eta}_{\gamma}, \\
\xi^{\alpha}= & (a / 2+i b) \theta^{\alpha}+k_{\gamma \delta} x_{+}^{\alpha \gamma} \theta^{\delta}+i \theta^{2} \eta^{\alpha}+x_{+}^{\alpha \beta} \bar{\eta}_{\beta}, \\
\bar{\xi}^{\alpha}= & (a / 2-i b) \bar{\theta}^{\alpha}+k_{\gamma \delta} x_{-}^{\alpha \gamma} \bar{\theta}^{\delta}+i \bar{\theta}^{2} \bar{\eta}^{\alpha}+x_{-}^{\alpha \beta} \eta_{\beta},
\end{aligned}
$$

where

$$
x_{ \pm}^{\alpha \beta}=x^{\alpha \beta} \pm 2 i \theta^{(\alpha} \bar{\theta}^{\beta)} .
$$

There is a superform $\xi$ associated with the superconformal Killing vector,

$$
\xi=\xi^{A} D_{A}=\frac{1}{2} \xi^{\alpha \beta}(z) \partial_{\alpha \beta}+\xi^{\alpha}(z) D_{\alpha}-\bar{\xi}^{\alpha}(z) \bar{D}_{\alpha},
$$

which obeys the following important relation

$$
\left[\xi, D_{\alpha}\right] \propto D_{\beta}
$$

Equation (B.11) means that the superconformal transformations respect the chirality and hence they can be extended to chiral superfields.

Either from (B.11) or from the explicit relations (B.6)-(B.8) one can deduce the following properties for spinor components of the superconformal Killing vector,

$$
\begin{aligned}
\xi^{\alpha} & =\frac{i}{6} \bar{D}_{\beta} \xi^{\alpha \beta}, & \bar{D}_{\alpha} \xi_{\beta}=0, \\
\bar{\xi}^{\alpha} & =-\frac{i}{6} D_{\beta} \xi^{\alpha \beta}, & D_{\alpha} \bar{\xi}_{\beta}=0,
\end{aligned}
$$

and for the vector one,

$$
D^{2} \xi^{\alpha \beta}=\bar{D}^{2} \xi^{\alpha \beta}=0, \quad D^{(\alpha} \xi^{\beta \gamma)}=\bar{D}^{(\alpha} \xi^{\beta \gamma)}=0
$$

The relations (B.12) show that $\xi^{\alpha}$ and $\bar{\xi}^{\alpha}$ are chiral and antichiral, respectively. Moreover, they are expressed in terms of the vector component $\xi^{\alpha \beta}$ which satisfies (B.13). In fact, the equations (B.13) are main defining relations for the superconformal Killing vector which lead to the standard equation for $\xi^{m}=\frac{1}{2} \gamma_{\alpha \beta}^{m} \xi^{\alpha \beta}$,

$$
\partial_{m} \xi_{n}+\partial_{n} \xi_{m}=\frac{2}{3} \eta_{m n} \partial_{p} \xi^{p} .
$$


As is shown in [11], one can in principle start with (B.11), then deduce (B.12) and (B.13) and after that derive (B.6) as a solution of (B.13). ${ }^{6}$

We will need also the following properties of the components of the superconformal Killing vector

$$
\begin{aligned}
D^{(\alpha} \xi^{\beta)}+\bar{D}^{(\alpha} \bar{\xi}^{\beta)} & =0, \\
D^{\alpha} \xi_{\alpha}-\bar{D}^{\alpha} \bar{\xi}_{\alpha} & =-\frac{1}{3} \partial_{\alpha \beta} \xi^{\alpha \beta}=-2 \rho,
\end{aligned}
$$

where

$$
\rho=a+k_{\alpha \beta} x^{\alpha \beta}+2 i \theta^{\alpha} \eta_{\alpha}+2 i \bar{\theta}^{\alpha} \bar{\eta}_{\alpha}
$$

The superfield $\rho$ obeys

$$
D_{\alpha} \rho=2 i\left(k_{\alpha \beta} \bar{\theta}^{\beta}+\eta_{\alpha}\right), \quad \bar{D}_{\alpha} \rho=-2 i\left(k_{\alpha \beta} \theta^{\beta}+\bar{\eta}_{\alpha}\right),
$$

while the following second derivatives of $\rho$ vanish

$$
D^{2} \rho=\bar{D}^{2} \rho=D^{\alpha} \bar{D}_{\alpha} \rho=0 .
$$

Let us introduce also the expressions

$$
\begin{aligned}
\sigma & =\frac{1}{4} \partial_{\alpha \beta} \xi^{\alpha \beta}-\frac{1}{2} D_{\alpha} \xi^{\alpha}=a-i b+k_{\alpha \beta} x_{+}^{\alpha \beta}+4 i \theta^{\alpha} \eta_{\alpha} \\
\bar{\sigma} & =\frac{1}{4} \partial_{\alpha \beta} \xi^{\alpha \beta}+\frac{1}{2} \bar{D}_{\alpha} \bar{\xi}^{\alpha}=a+i b+k_{\alpha \beta} x_{-}^{\alpha \beta}+4 i \bar{\theta}^{\alpha} \bar{\eta}_{\alpha} .
\end{aligned}
$$

These superfields are chiral and antichiral, respectively,

$$
\bar{D}_{\alpha} \sigma=0, \quad D_{\alpha} \bar{\sigma}=0 .
$$

Clearly, the parameters $\sigma, \bar{\sigma}$ and $\rho$ are related to each other,

$$
\rho=\frac{1}{2}(\sigma+\bar{\sigma})
$$

Now we consider a representation of the superconformal group on superfields in the $\mathcal{N}=2$ superspace. Given a (real) superfield $V$ defined in full $\mathcal{N}=2$ superspace with mass-dimension $l$, we define its infinitesimal superconformal transformation as

$$
\delta_{\mathrm{sc}} V=(l \rho+\xi) V,
$$

where $\xi$ and $\rho$ are defined in (B.10) and (B.17), respectively. Analogously, the superfields $\sigma$ and $\bar{\sigma}$ given by (B.20,B.21) are used to define the superconformal transformations for a chiral $Q$ and an antichiral $\bar{Q}$ superfields of mass dimension $l$,

$$
\delta_{\mathrm{sc}} Q=(l \sigma+\xi) Q, \quad \delta_{\mathrm{sc}} \bar{Q}=(l \bar{\sigma}+\xi) \bar{Q} .
$$

\footnotetext{
${ }^{6}$ Note that (B.6) is not the general solution of (B.13). The general solution involves also the parameters of usual translations, Lorentz transformations and supertranslations. However we omit here these parameters since the superspace approach provides the covariance of all considered actions under super Poincaré group.
} 
The superfields which transform under the superconformal group by the rules (B.24,B.25) are usually referred to as the quasi-primary superfields with scaling dimension $l$.

An action $S=\int d^{3} x d^{4} \theta \mathcal{L}$ is superconformal if the Lagrangian $\mathcal{L}$ transforms as a quasi-primary scalar superfield with the scaling dimension $l=+1$, i.e.,

$$
\delta_{\mathrm{sc}} \mathcal{L}=(\rho+\xi) \mathcal{L} \quad \Rightarrow \quad \delta_{\mathrm{sc}} S=0
$$

However, this is not the necessary and sufficient condition of superconformal invariance since more generally the Lagrangian can transform as

$$
\delta_{\mathrm{sc}} \mathcal{L}=(\rho+\xi) \mathcal{L}+\sigma \mathcal{K}+\bar{\sigma} \overline{\mathcal{K}} \quad \Leftrightarrow \quad \delta_{\mathrm{sc}} S=0
$$

where $\mathcal{K}$ and $\overline{\mathcal{K}}$ are linear functions,

$$
\bar{D}^{2} \mathcal{K}=0, \quad D^{2} \overline{\mathcal{K}}=0 .
$$

These additional contributions with $\mathcal{K}$ and $\overline{\mathcal{K}}$ in the variation of the Lagrangian (B.27) do not break the invariance of the action since they vanish in passing from the full to the (anti)chiral superspace.

Similarly, the superconformal invariance of an action in the chiral superspace $S_{c}=$ $\int d^{3} x d^{2} \theta \mathcal{L}_{c}$ is guaranteed if the Lagrangian $\mathcal{L}_{c}$ is a chiral quasi-primary superfield with the scaling dimension $l=+2$,

$$
\delta_{\mathrm{sc}} \mathcal{L}_{c}=(2 \sigma+\xi) \mathcal{L}_{c} \quad \Leftrightarrow \quad \delta_{\mathrm{sc}} S_{c}=0
$$

\section{References}

[1] J. Bagger, N. Lambert, Modeling multiple M2's, Phys. Rev. D75 (2007) 045020, hep-th/0611108;

Gauge symmetry and supersymmetry of multiple M2-branes, Phys. Rev. D77 (2008) 065008, arXiv:0711.0955 [hep-th];

Comments on multiple M2-branes, JHEP 0802 (2008) 105, arXiv:0712.3738 [hep-th];

Three-algebras and N=6 Chern-Simons gauge theories, Phys. Rev. D79 (2009) 025002, arXiv:0807.0163 [hep-th];

A. Gustavsson, Algebraic structures on parallel M2-branes, Nucl. Phys. B811 (2009) 66, arXiv:0709.1260 [hep-th];

Selfdual strings and loop space Nahm equations, JHEP 0804 (2008) 083, arXiv:0802.3456 [hep-th].

[2] O. Aharony, O. Bergman, D.L. Jafferis, J. Maldacena, $N=6$ superconformal ChernSimons-matter theories, M2-branes and their gravity duals, JHEP 0810 (2008) 091, arXiv:0806.1218 [hep-th]. 
[3] A.N. Redlich, Gauge noninvariance and parity violation of three-dimensional fermions, Phys. Rev. Lett. 52 (1984) 18;

Parity violation and gauge noninvariance of the effective gauge field action in three dimensions, Phys. Rev. D29 (1984) 2366.

[4] T. Ohrndorf, The effective Lagrangian of supersymmetric Yang-Mills theory, Phys. Lett. B176 (1986) 421;

I.N. McArthur, T.D. Gargett, A 'Gaussian' approach to computing supersymmetric effective actions, Nucl. Phys. B497 (1997) 525, hep-th/9705200;

N.G. Pletnev, A.T. Banin, Covariant technique of derivative expansion of one loop effective action, Phys. Rev. D60 (1999) 105017, hep-th/9811031.

[5] S.M. Kuzenko, I.N. McArthur, Low-energy dynamics in N=2 super QED: Two loop approximation, JHEP 0310 (2003) 029, hep-th/0308136.

[6] S.M. Kuzenko, S.J. Tyler, Supersymmetric Euler-Heisenberg effective action: Twoloop results, JHEP 0705 (2007) 081, hep-th/0703269.

[7] N.J. Hitchin, A. Karlhede, U. Lindström, M. Roček, Hyperkahler metrics and supersymmetry, Commun. Math. Phys. 108 (1987) 535.

[8] B.M. Zupnik, D.G. Pak, Superfield formulation of the simplest three-dimensional gauge theories and conformal supergravities, Theor. Math. Phys. 77 (1988) 1070 [Teor. Mat. Fiz. 77 (1988) 97];

Topologically massive gauge theories in superspace, Sov. Phys. J. 31 (1988) 962.

[9] S.J. Gates Jr., H. Nishino, Remarks on N=2 supersymmetric Chern-Simons theories, Phys. Lett. B281 (1992) 72.

[10] I.L. Buchbinder, S.M. Kuzenko, A.A. Tseytlin, On low-energy effective actions in N=2, N=4 superconformal theories in four-dimensions, Phys. Rev. D62 (2000) 045001, hep-th/9911221.

[11] J.-H. Park, Superconformal symmetry in three-dimensions, J. Math. Phys. 41 (2000) 7129, hep-th/9910199.

[12] I.L. Buchbinder, S.M. Kuzenko, Ideas and Methods of Supersymmetry and Supergravity, IOP Publishing, Bristol and Philadelphia, 1998, 656 p.

[13] E.A. Ivanov, Chern-Simons matter systems with manifest N=2 supersymmetry, Phys. Lett. B268 (1991) 203.

[14] H. Osborn, $N=1$ superconformal symmetry in four-dimensional quantum field theory, Annals Phys. 272 (1999) 243, hep-th/9808041.

[15] J.-H. Park, N=1 superconformal symmetry in four-dimensions, Int. J. Mod. Phys. A13 (1998) 1743, hep-th/9703191;

Superconformal symmetry and correlation functions, Nucl. Phys. B559 (1999) 455, hep-th/9903230. 
[16] S.M. Kuzenko, I.N. McArthur, On the background field method beyond one loop: A Manifestly covariant derivative expansion in super Yang-Mills theories, JHEP 0305 (2003) 015, hep-th/0302205.

[17] A.J. Niemi, G.W. Semenoff, Axial-anomaly-induced fermion fractionization and effective gauge-theory actions in odd-dimensional space-times, Phys. Rev. Lett. 51 (1983) 2077.

[18] G.V. Dunne, Aspects of Chern-Simons theory, hep-th/9902115.

[19] S.J. Gates, M.T. Grisaru, M. Roček, W. Siegel, Superspace or One Thousand and One Lessons in Supersymmetry, Benjamin/Cummings, Reading, MA, 1983, 548 p.

[20] O. Aharony, A. Hanany, K.A. Intriligator, N. Seiberg, M.J. Strassler, Aspects of N=2 supersymmetric gauge theories in three-dimensions, Nucl. Phys. B499 (1997) 67, hep-th/9703110.

[21] E.W. Weisstein, CRC Concise Encyclopedia of Mathematics, Chapman \& Hall/CRC, 2003.

[22] I.L. Buchbinder, E.A. Ivanov, O. Lechtenfeld, N.G. Pletnev, I.B. Samsonov, B.M. Zupnik, Quantum N=3, d=3 Chern-Simons matter theories in harmonic superspace, JHEP 10 (2009) 075, arXiv:0909.2970 [hep-th].

[23] B. de Witt, M. Roček, Improved tensor multiplets, Phys. Lett. B109 (1982) 439;

U. Lindström, M. Roček, Scalar tensor duality and N=1, N=2 nonlinear sigma models, Nucl. Phys. B222 (1983) 285.

[24] S.M. Kuzenko, U. Lindstrom, R. von Unge, New extended superconformal sigma models and quaternion Kähler manifolds, JHEP 0909 (2009) 119, arXiv:0906.4393 [hep-th].

[25] E. Koh, S. Lee, S. Lee, Topological Chern-Simons sigma model, JHEP 0909 (2009) 122, arXiv:0907.1641 [hep-th].

[26] D. Gaiotto, E. Witten, Janus configurations, Chern-Simons couplings, and the thetaAngle in N=4 super Yang-Mills theory, arXiv:0804.2907 [hep-th].

[27] K.A. Intriligator, N. Seiberg, Mirror symmetry in three-dimensional gauge theories, Phys. Lett. B387 (1996) 513, hep-th/9607207;

N. Seiberg, E. Witten, Gauge dynamics and compactification to three-dimensions, in *Saclay 1996, The mathematical beauty of physics*, 333-366, hep-th/9607163;

A. Hanany, E. Witten, Type IIB superstrings, BPS monopoles, and three-dimensional gauge dynamics, Nucl. Phys. B492 (1997) 152, hep-th/9611230.

[28] J. de Boer, K. Hori, Y. Oz, Dynamics of N=2 supersymmetric gauge theories in three-dimensions, Nucl. Phys. B500 (1997) 163, hep-th/9703100;

J. de Boer, K. Hori, Y. Oz, Z. Yin, Branes and mirror symmetry in N=2 supersymmetric gauge theories in three-dimensions, Nucl. Phys. B502 (1997) 107, hep-th/9702154;

J. de Boer, K. Hori, H. Ooguri, Y. Oz, Mirror symmetry in three-dimensional gauge theories, quivers and D-branes, Nucl. Phys. B493 (1997) 101, hep-th/9611063. 
[29] D.L. Jafferis, X. Yin, Chern-Simons-matter theory and mirror symmetry, arXiv:0810.1243;

K. Jensen, A. Karch, ABJM mirrors and a duality of dualities, JHEP 0909 (2009) 004, arXiv:0906.3013 [hep-th];

D.L. Jafferis, Quantum corrections to $N=2$ Chern-Simons theories with flavor and their AdS4 duals arXiv:0911.4324 [hep-th].

[30] O. Aharony, O. Bergman, D.L. Jafferis, Fractional M2-branes, JHEP 0811 (2008) 043, arXiv:0807.4924 [hep-th];

M. Schnabl, Y. Tachikawa, Classification of $N=6$ superconformal theories of ABJM type, arXiv:0807.1102 [hep-th];

D. Martelli, J. Sparks, Moduli spaces of Chern-Simons quiver gauge theories and AdS(4)/CFT(3), Phys. Rev. D78 (2008) 126005, arXiv:0808.0912 [hep-th];

A. Hanany, A. Zaffaroni, Tilings, Chern-Simons theories and M2 branes, JHEP 0810 (2008) 111, arXiv:0808.1244 [hep-th];

S. Franco, A. Hanany, J. Park, D. Rodriguez-Gomez, Towards M2-brane theories for generic toric singularities, JHEP 0812 (2008) 110, arXiv:0809.3237 [hep-th];

A. Hanany, Y.-H. He, M2-branes and quiver Chern-Simons: A taxonomic study, arXiv:0811.4044 [hep-th];

E. Imeroni, On deformed gauge theories and their string/M-theory duals, JHEP 0810 (2008) 026, arXiv:0808.1271 [hep-th];

D.L. Jafferis, A. Tomasiello, A simple class of $N=3$ gauge/gravity duals, JHEP 0810 (2008) 101, arXiv:0808.0864 [hep-th];

D. Gaiotto, A. Tomasiello, The gauge dual of Romans mass, JHEP 1001 (2010) 015, arXiv:0901.0969 [hep-th].

[31] N. Akerblom, C. Saemann, M. Wolf, Marginal deformations and 3-algebra structures, Nucl. Phys. B826 (2010) 456, arXiv:0906.1705 [hep-th].

[32] M.S. Bianchi, S. Penati, M. Siani, Infrared stability of N=2 Chern-Simons matter theories, arXiv:0912.4282 [hep-th];

Infrared stability of ABJ-like theories, JHEP 1001 (2010) 080, arXiv:0910.5200 [hep-th].

[33] L.V. Avdeev, G.V. Grigoryev, D.I. Kazakov, Renormalizations in abelian ChernSimons field theories with matter, Nucl. Phys. B382 (1992) 561;

L.V. Avdeev, D.I. Kazakov, I.N. Kondrashuk, Renormalizations in supersymmetric and nonsupersymmetric non-abelian Chern-Simons field theories with matter, Nucl. Phys. B391 (1993) 333.

[34] I.L. Buchbinder, E.A. Ivanov, O. Lechtenfeld, N.G. Pletnev, I.B. Samsonov, B.M. Zupnik, ABJM models in N=3 harmonic superspace, JHEP 03 (2009) 096, arXiv:0811.4774 [hep-th]. 